\title{
Application of the GeoSequencing Module to ensure optimised underground mine schedules with reduced geotechnical risk
}

\author{
R Hauta MIRARCO Mining Innovation, Canada \\ M Whittier MIRARCO Mining Innovation, Canada \\ L Fava MIRARCO Mining Innovation, Canada
}

\begin{abstract}
Conventionally, engineers will impose stope sequencing rules in order to ensure the stability of excavations as mining progresses. Typical rules include fixed direction, primary-secondary and chevron. Using a mine planning software suite, a mine layout is designed, and a set of stope-to-stope precedence links are created, enforcing the applicable rule. The mine planner produces a schedule that meets these and other constraints, in an effort to achieve a high net present value (NPV) for the operation. This time-consuming process limits the planner's ability to assess alternative mining strategies. Further, the process tends to over-constrain the mine schedule, since it is often the case that an alternative set of precedence constraints can enforce the same rule. By over-constraining the schedule, and by not assessing alternative strategies, significant value may be lost.

The GeoSequencing Module is software that facilitates the assessment of stope sequencing strategies, through integration with the Schedule Optimization Tool (SOT). The GeoSequencing Module rapidly generates multiple alternative sets of stope-to-stope precedence links that enforce stope sequencing rules selected by the user. Each set of precedence links is referred to as a geosequencing scenario. For each geosequencing scenario, SOT optimises the life-of-mine schedule, allowing the planner to identify the scenario that supports the highest value for the mining operation. Furthermore, the software has been integrated with a boundary element solver, facilitating assessment of the geotechnical stability of the optimised schedules.

A case study for an underground mine has been conducted to validate this methodology. The study demonstrates that multiple mining strategies can be conveniently assessed to determine which scenario yields the most desirable outcome for the mining operation in terms of both geotechnical stability and financial assessment.
\end{abstract}

Keywords: mine planning, mine schedule optimisation, geomechanics, stope sequencing, numerical modelling

\section{Introduction}

Conventional long-term mine planning practices often restrict the planner's ability to assess multiple alternative mine planning strategies. Ideally, multiple constraints and parameters should be taken into account, such as ventilation requirements, product price and ore grade uncertainty, as well as geotechnical extraction constraints. Unoptimised plans are often produced during different stages of mine planning due to the time-consuming nature of developing and assessing alternative extraction strategies.

The Schedule Optimization Tool (SOT) can assist a planner in addressing these challenges. SOT optimises the net present value (NPV) of life-of-mine schedules. It uses heuristics and a custom genetic algorithm to rapidly generate, assess and optimise mine schedules which conform to all user-defined operational and precedence constraints (Maybee et al. 2010; Fava et al. 2011).

Three new software modules that integrate with SOT have been developed through MIRARCO's SOT+ research project, to enhance the planner's ability to constrain, optimise, and analyse mine schedules in order to extract the maximum value from a mining operation. A high-level description of these software modules follows. 
Advanced Valuation: Schedules are optimised while accounting for product price distributions and product grade confidence distributions. Two methodologies are implemented, aiming to provide long-term schedules that are optimised and robust in the context of uncertainty.

Ventilation Constraint Module: The Ventilation Constraint Module is integrated with a ventilation network solver to support simultaneous optimisation of the life-of-mine schedule and the ventilation system. From the available airflows provided by the network solver, the software automatically generates ventilation-based equipment constraints.

GeoSequencing: The GeoSequencing Module rapidly generates precedence constraints between stopes to implement user-selected rules motivated by geotechnical constraints. This tool is the focus of this paper.

\subsection{GeoSequencing Module motivation and description}

A mine layout prepared in general mine planning (GMP) software consists of a set of mining activities (or tasks) with corresponding dependencies (or precedence constraints) linking them. The mining activities include, for example, ramps, raises, drifts and stopes. The dependencies constrain the order in which the activities may be scheduled; for example, they may represent physical adjacencies. Figure 1 illustrates activities and dependencies in computer-aided design (CAD) software; such dependencies are normally created between task polygon centroids using a spatial search criterion, or based on task attributes. Attributes may include, for example, name, tonnage, length, product grade and rate.

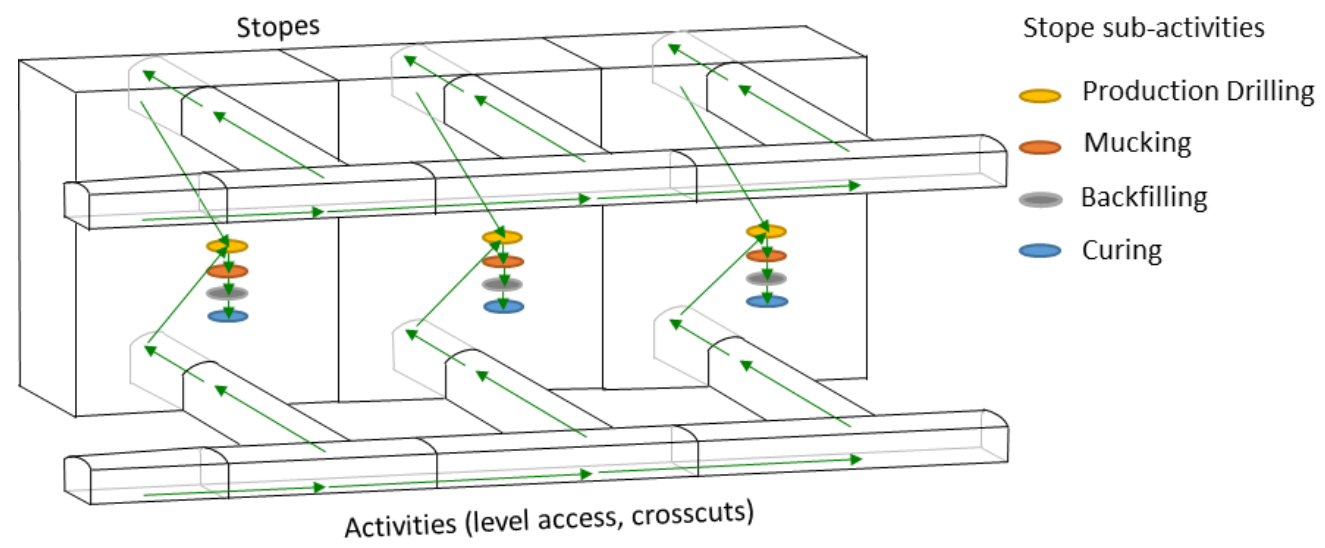

Figure 1 Mine development and stope tasks with finish-start dependencies (green arrows)

The primary purpose of defining dependencies is to achieve and enforce physical and practical planning constraints. Because of the large quantity of dependencies that are generated in mine designs, filtering and modifying stope-to-stope dependencies becomes difficult (Figure 2).

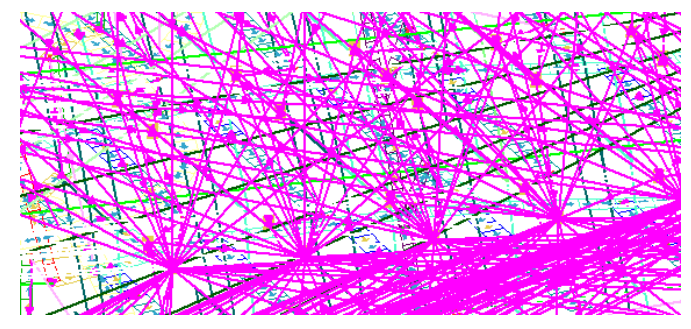

Figure 2 The set of planning dependencies in this project has become complex, making the process of modifying and troubleshooting links challenging and tedious

Dependencies are often added to enforce a stoping pattern selected by geotechnical experts in order to ensure geotechnical stability. However, there are two problems that may be associated with this conventional approach. Mine planners may unintentionally over-constrain schedules when defining these dependencies. Often, a given stoping pattern will have a variety of possible dependency implementations (i.e. alternative sets of dependencies) that all achieve the same extraction strategy. For example, when 
implementing a primary-secondary stoping pattern, a selection is often made at the outset as to which stopes will be the primary stopes, rather than considering alternative candidate primary stopes. Secondly, it is prohibitively time-consuming to implement any identified alternative stoping patterns. As a result, alternative strategies that have the potential to lead to more desirable initial cash flows or higher NPV schedules tend not to be assessed.

The GeoSequencing Module has been developed to address these issues by facilitating the efficient application and assessment of alternative stope extraction scenarios. The software rapidly generates multiple alternative sets of stope-to-stope precedence links that enforce stope sequencing rules selected by the user. Each set of precedence links is referred to as a 'geosequencing scenario'.

We introduce here some terminology, since it varies regionally. The stoping rule 'primary-secondary' describes a pattern where stopes on a level are alternately flagged as primary or secondary, in alignment with adjacent levels. Temporary pillars are created by mining out primary stopes before secondary stopes. The number of 'lifts' refers to the maximum number of primary stopes that can advance vertically before any secondary stopes on the current level are mined. Another stoping rule is 'chevron', which imposes a centre-out pillarless mining stoping pattern, as shown in Figure 3. A third stoping rule referred to in this study is 'fixed mining direction', which simply indicates that stopes must be excavated in a given direction, such as west-to-east. 'Panel' refers to vertically aligned stopes; for example, in Figure 3, nine panels are shown.

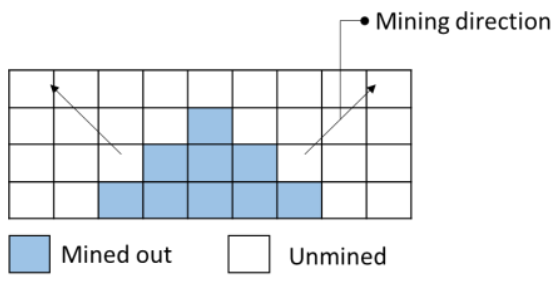

Figure 3 Chevron mining pattern (after Morrison 1996)

The set of rules that are available in the GeoSequencing Module, some of which were used in the case study, are shown in Table 1, along with the associated parameters for each.

Table 1 Common geotechnically motivated rules (stoping patterns)

\begin{tabular}{lll}
\hline Rule & Directions & Parameters \\
\hline Primary-secondary & Bottom-up, top-down & $\begin{array}{l}\text { Maximum and minimum } \\
\text { number of lifts }\end{array}$ \\
$\begin{array}{l}\text { Chevron (also known as } \\
\text { pyramid) }\end{array}$ & Bottom-up, top-down & $\begin{array}{l}\text { Maximum and minimum } \\
\text { number of lifts }\end{array}$ \\
$\begin{array}{l}\text { Primary-secondary, chevron } \\
\text { Fixed mining direction }\end{array}$ & Bottom-up, top-down & $\begin{array}{l}\text { Maximum and minimum } \\
\text { number of lifts for primary } \\
\text { and secondary stopes }\end{array}$ \\
& Positive or negative $x, y, z$ & $\begin{array}{l}\text { Maximum and minimum } \\
\text { number of panels }\end{array}$ \\
\hline
\end{tabular}




\section{Geosequencing case study}

\subsection{Mine layout}

A case study was conducted applying the GeoSequencing Module to the mine design shown in Figure 4. This mine has 291 stopes, and uses a sublevel open stoping mining method with both longitudinal and transverse designs.

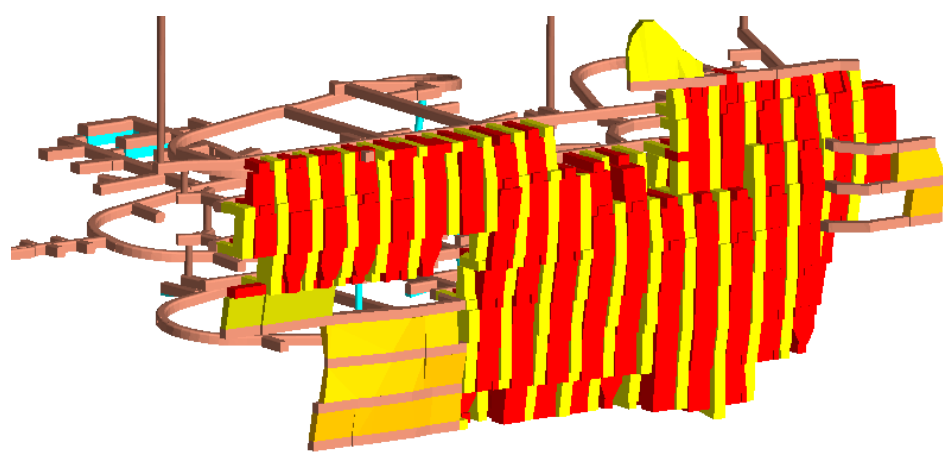

Figure 4 Mine layout for the validation case study

\section{$2.2 \quad$ Methodology}

The workflow for the case study, shown in Figure 5, makes use of GMP software, the GeoSequencing Module (MIRARCO Mining Innovation 2017), SOT (Revolution Mining Software, Inc. 2017) and Map3D (Wiles 2017). These software tools have been loosely coupled to facilitate this process. The GMP software is used to create the mine layout, the GeoSequencing Module is used to create geosequencing scenarios, and SOT is used to generate optimised life-of-mine schedules for each scenario. Map3D, a boundary element method (BEM) solver, is used to assess the geotechnical stability of the optimised schedules. The results can be reviewed in the GMP software and in Map3D.

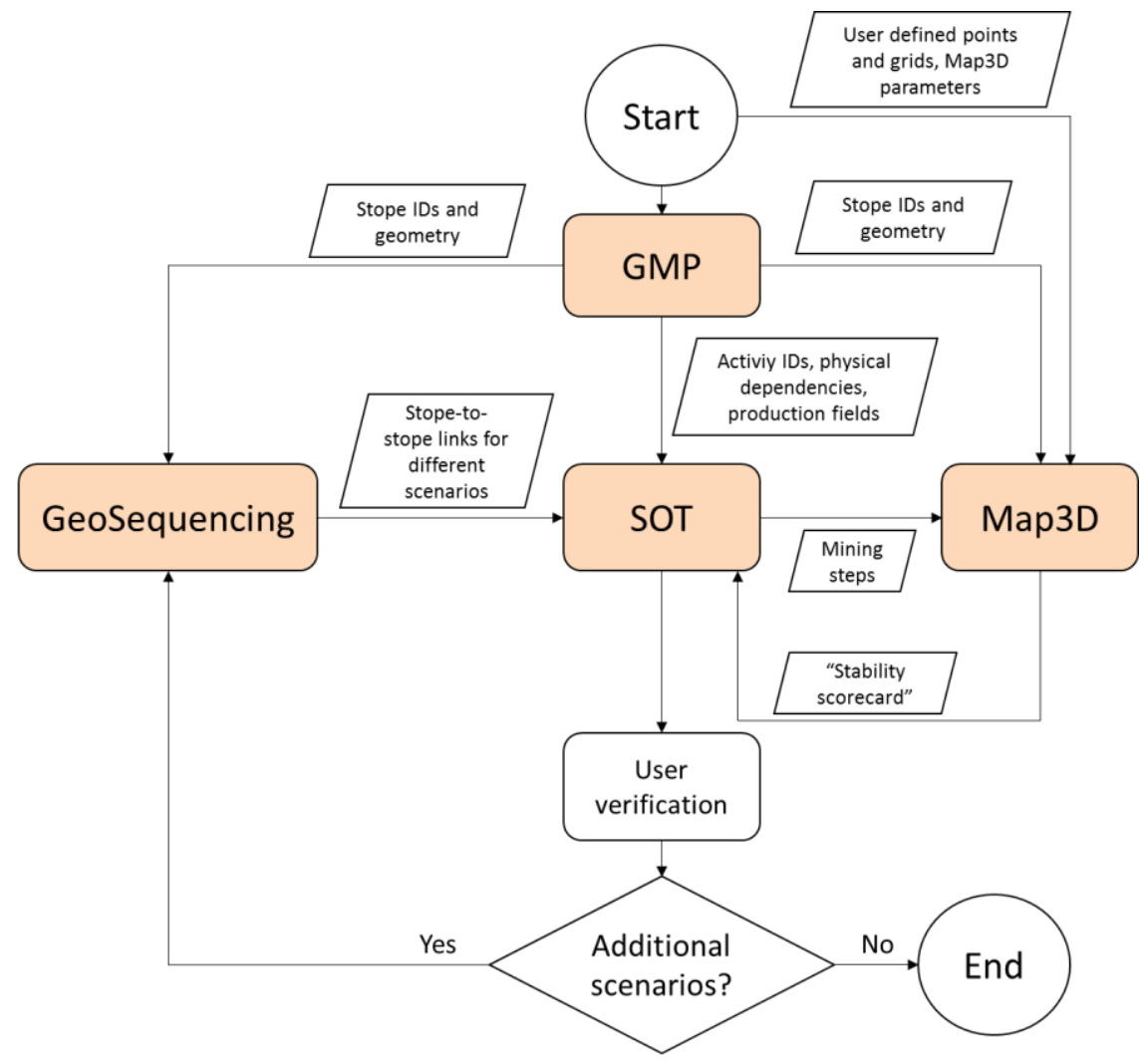

Figure 5 Workflow for generating and assessing alternative geosequencing scenarios 


\subsubsection{Generating geosequencing scenarios}

Stope design entities were imported as $x, y, z$ coordinates into the GeoSequencing Module from the GMP software. Taking threshold distance parameters as input, the GeoSequencing Module calculated stope adjacencies (or neighbour relationships) by means of a unique ray tracing and grid method. The software also identified connected sets of stopes; the majority of stopes (270 of 291) were assigned to one connected set. From defined thresholds and stope attributes, multiple interconnected sets of stopes were generated. Rules were applied within and between the connected sets of stopes. Compatible combinations of stoping rules were selected for application to the largest connected set, as given in Table 2. The other connected sets had logical rules applied to them, such as fixed mining direction.

Table 2 Geosequencing rule scenarios applied for the case study

\begin{tabular}{ll}
\hline Generated geosequencing scenarios \\
\hline 1 & Primary-secondary, bottom-up (red stopes as primary) \\
2 & Primary-secondary, bottom-up (yellow stopes as primary) \\
3 & Chevron, bottom-up \\
4 & Primary-secondary, chevron, bottom-up (red stopes as primary) \\
5 & Primary-secondary, chevron, bottom-up (yellow stopes as primary) \\
\hline
\end{tabular}

Several geosequencing scenarios were generated. Alternative scenarios were generated for each primary-secondary rule by changing the designation of primary and secondary stopes.

For the primary-secondary-chevron scenarios, no maximum number of lifts was imposed. These became test cases expected to result in reduced geotechnical stability. For each geosequencing scenario, dependencies generated by the software were verified manually in the GeoSequencing Module by selecting a stope and its predecessors and successors, which were visualised as shown in Figure 6.

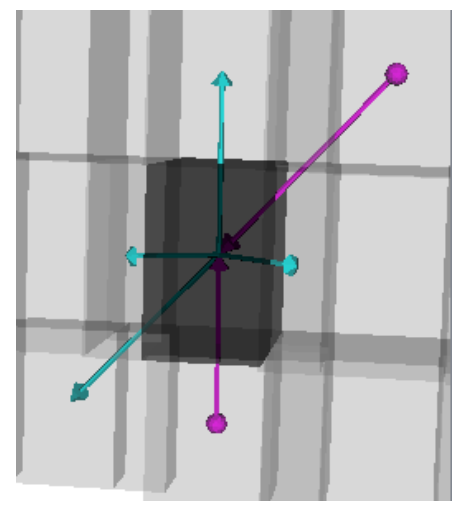

Figure 6 Visual verification of stope-to-stope dependencies - the selected stope's predecessors are indicated in magenta, and its successors are indicated in cyan

The five scenarios generated by the GeoSequencing Module were imported into SOT. The same operational resource constraints and financial model were used for all scenarios. For each scenario, SOT then generated life-of-mine schedules of stoping and development activities with the objective of maximising NPV. 


\subsubsection{Assessing schedule stability}

The CAD entities and activity identifiers were imported into Map3D, and pre-mining stress, rockmass and backfill parameters provided by the mining company were configured in Map3D for the stability assessment. The following parameters were also manually defined:

- Material properties.

- Principal stresses.

- Grids and grid discretisation parameters.

- Points at which the stability is to be assessed for each mining step ( $>4,000$ such points).

The SOT stoping schedule was then imported into Map3D, in the form of corresponding 'mining steps' at intervals of one year for the entire mine life. Differential stress $\left(\sigma_{D}\right)$, which is the difference between the major and minor principal stresses $\left(\sigma_{1}-\sigma_{3}\right)$, was selected as the stability metric for these assessments, since it is a common measure of mining-induced stress (May 2014; Shnorhokian et al. 2014). Map3D calculated the differential stress at the defined points surrounding the stopes, for each mining step. A schedule 'stability scorecard' was generated by the GeoSequencing Module for each SOT schedule based on the aggregated results from modelling analysis. Specifically, the stability score was the average differential stress over all points for each mining step. The schedules, and therefore the rules (stoping patterns), resulting in increased stability over alternative options were then manually assessed.

\subsection{Results and discussion}

The optimised life-of-mine NPV for each geosequencing scenario is presented in Table 3 . The difference between the best and worst case scenarios, in terms of their life-of-mine NPV, is only USD 8.9 M. From a project scale standpoint, this difference is fairly insignificant, especially considering the uncertain effect that the time value of money can have on a project when it is actually realised.

Table 3 SOT-optimised NPVs for the five geosequencing scenarios

\begin{tabular}{lll}
\hline Geosequencing scenario & NPV (M) \\
\hline 1 & Primary-secondary, bottom-up (red stopes as primary) & USD 426.5 \\
2 & Primary-secondary, bottom-up (yellow stopes as primary) & USD 419.3 \\
3 & Chevron, bottom-up & USD 417.6 \\
4 & Primary-secondary, chevron, bottom-up (red stopes as primary) & USD 425.9 \\
5 & Primary-secondary, chevron, bottom-up (yellow stopes as primary) & USD 424.5 \\
\hline
\end{tabular}

However, even with similar life-of-mine NPVs, differences in cash flows between scenarios can be non-negligible. The cash flows for each geosequencing scenario in years four and five are notably different, as depicted in Figure 7, where the cumulative NPV has been plotted. The difference between Scenarios 1 and 5 in year four is USD $63.8 \mathrm{M}$, while the difference between Scenarios 1 and 3 in year five is USD $41.3 \mathrm{M}$. Likewise, the worst scenario from a financial standpoint differs depending on the period being observed and the annual capital budget of the operation. These financial differences can be considered significant and clearly demonstrate the effect that different geosequencing scenarios can have on the cash flow and subsequent financial feasibility of a project. For some operations, such differences in capital requirements can be the difference between a project being considered viable or unfeasible. 


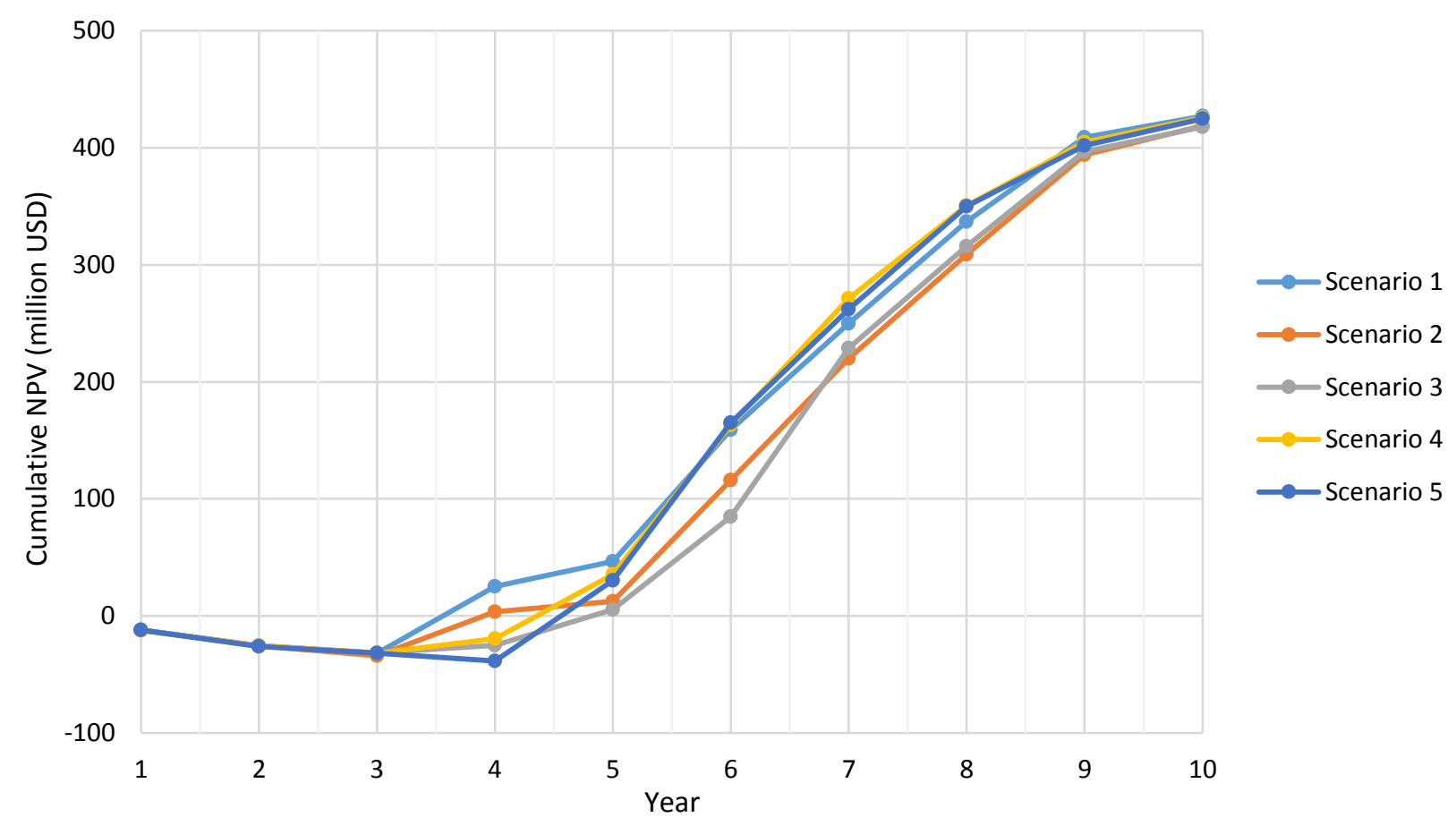

Figure 7 Annual cumulative NPV for each geosequencing scenario

The average $\sigma_{D}$ was also calculated for each optimised schedule, and the resulting stability scorecard is shown in Table 4.

Table 4 Results of stability analysis for the five geosequencing scenarios

\begin{tabular}{|c|c|c|c|c|c|c|c|c|c|}
\hline \multirow{2}{*}{\multicolumn{2}{|c|}{ Geosequencing scenario }} & \multicolumn{8}{|c|}{ Average $\sigma_{D}$} \\
\hline & & Year 1 & Year 2 & Year 3 & Year 4 & Year 5 & Year 6 & Year 7 & Year 8 \\
\hline 1 & $\begin{array}{l}\text { Primary-secondary, bottom-up } \\
\text { (red stopes primary) }\end{array}$ & 7.2 & 7.4 & 7.6 & 8.1 & 8.7 & 9.2 & 9.5 & 9.4 \\
\hline 2 & $\begin{array}{l}\text { Primary-secondary, bottom-up } \\
\text { (yellow stopes primary) }\end{array}$ & 7.2 & 7.4 & 7.6 & 8.1 & 8.4 & 9.1 & 9.4 & 9.4 \\
\hline 3 & Chevron, bottom-up & 7.2 & 7.5 & 7.8 & 8.3 & 8.8 & 9.3 & 9.5 & 9.4 \\
\hline 4 & $\begin{array}{l}\text { Primary-secondary, chevron, } \\
\text { bottom-up (red stopes primary) }\end{array}$ & 7.2 & 7.4 & 8.1 & 9.0 & 9.3 & 9.3 & 9.5 & 9.5 \\
\hline 5 & $\begin{array}{l}\text { Primary-secondary, chevron, } \\
\text { bottom-up (yellow stopes } \\
\text { primary) }\end{array}$ & 7.2 & 7.8 & 8.2 & 8.5 & 9.2 & 9.4 & 9.5 & 9.5 \\
\hline
\end{tabular}

The stability values for each scenario are shown in Figure 8. As expected, the results of the study show that the primary-secondary chevron stoping patterns (Scenarios 4 and 5) with no constraints on the maximum number of lifts have poorer stability scores along with cumulative NPVs that differ considerably from other scenarios. These schedules could be less stable over the course of the mine life since the average $\sigma_{D}$ is higher than other mining sequences for the majority of the mine life. 


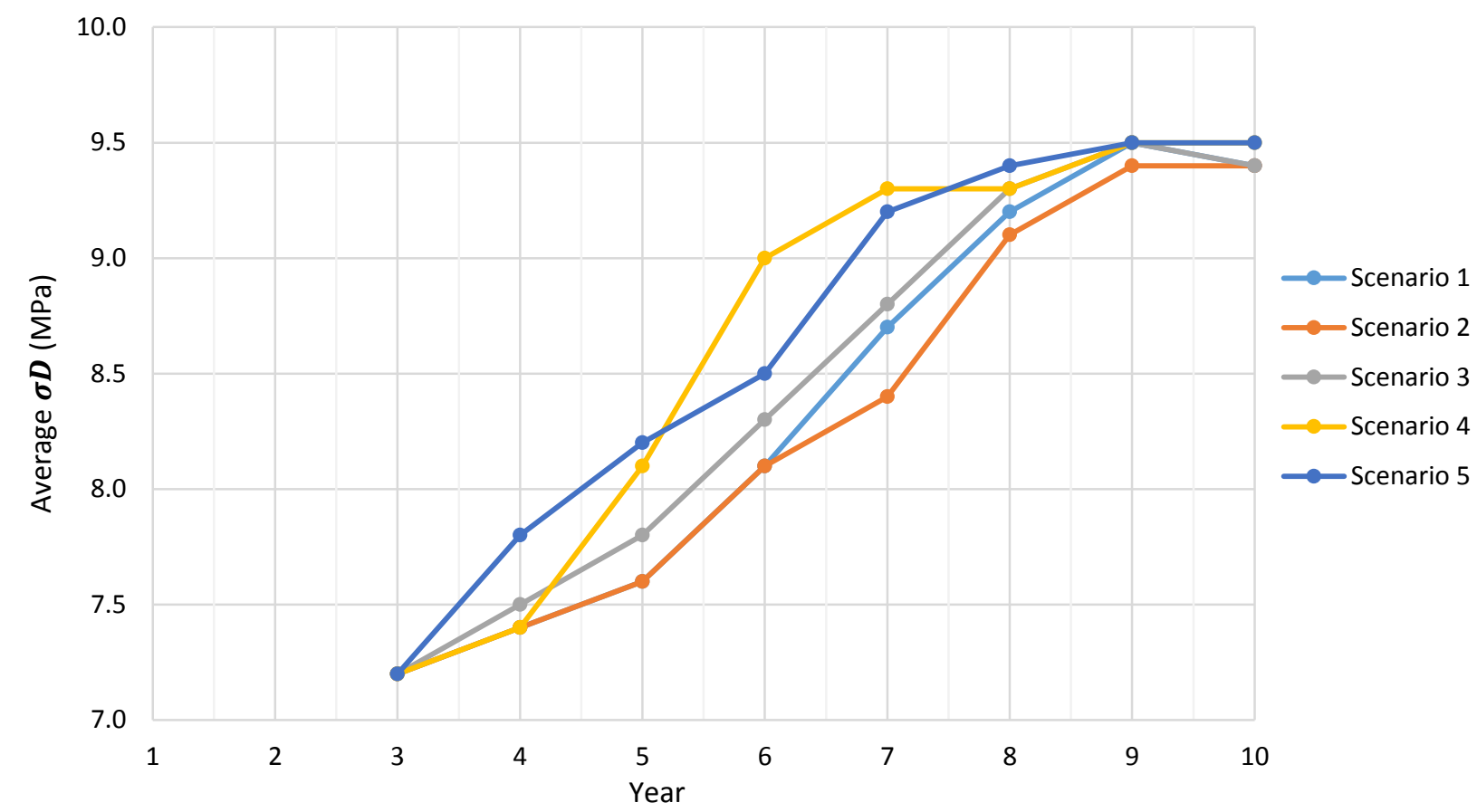

Figure 8 Annual average $\sigma_{D}$ for each geosequencing scenario, for each year in which stoping occurs

The results indicate that the implementation of geotechnical stoping rules can have an impact on the cash flow of the project. If a conventional process is employed, it is likely that fewer scenarios will be considered, and financially feasible, more stable scenarios may not be discovered.

Long-term stability assessment coupled with schedule optimisation should be integrated into the mine planning process to reduce the chance of instability-driven changes to the mine plan. Without this, dealing with unanticipated stability issues can result in operational cost overruns due to, for example, rehabilitation of mine workings. Mines may fail to meet their production targets, or even experience localised production shutdowns when geotechnical risks are not adequately managed. Additionally, major and insufficiently planned changes to the mine plan, such as being forced to change stope sequencing strategy, can significantly reduce the ability of a mining operation to achieve its anticipated economic value or meet its annual capital demands.

\section{$3 \quad$ Future work}

The set of rules and accompanying parameters implemented in the GeoSequencing Module will be expanded as required by future case studies. Alternative aggregations of stability values provided by commercial analysis tools such as Map3D will be investigated.

A validation exercise based on historical data from a mining operation - the schedule of completed and ongoing activities, and the mine's geotechnical record and related costs - would be valuable. Such a study would assist with devising evidence-based best practices for the mining operation.

\section{Conclusion}

The GeoSequencing Module facilitates the generation of alternative mine schedules adhering to geotechnically motivated stoping patterns without over-constraining the NPV optimisation. The workflow described allows the planner to select a long-term schedule based on simultaneous consideration of NPV, cash flows and geotechnical stability. 


\section{Acknowledgement}

The authors thank Newmont, Vale Canada Limited, Agnico Eagle Mines Limited, Deswik, Datamine, Dr Terry Wiles from Map3D, and the Ultra-Deep Mining Network (UDMN) at the Centre for Excellence in Mining Innovation (CEMI) for their technical and financial contributions to the SOT+ project. The invaluable work of Darren Janeczek, Scott McGarvey, and Chris Mangiardi of MIRARCO is gratefully acknowledged.

\section{References}

Fava, L, Millar, D \& Maybee, B 2011, 'Scenario evaluation through mine schedule optimisation', in R Kuyvenhoven, E Rubio \& M Smith (eds), Proceedings of the 2nd International Seminar on Mine Planning, Gecamin, Santiago, pp. 1-10.

May, W 2014, An Investigation of Induced Rock Stress and Related Damage in Popular Stope Sequencing Options Using Numerical Modelling, CSMM152 - dissertation project, Camborne School of Mines, Penryn.

Maybee, B, Fava, L, Dunn, P, Wilson, S \& Fitzgerald, J 2010, 'Toward optimum value in underground mine scheduling', CIM Journal, vol. 1, no. 3, pp. 176-182.

MIRARCO Mining Innovation 2017, SOT+, MIRARCO Mining Innovation, Sudbury, viewed 5 July 2017, http://www.mirarco.org/ sotplus/

Morrison, DM 1996, 'Deep hardrock mining: the future', CIM Bulletin, vol. 89, no. 1000, pp. 46-51.

Revolution Mining Software Inc. 2017, Schedule Optimization Tool, version 2.0, Revolution Mining Software Inc., viewed 5 July 2017 , http://www.revolutionmining.com/sot-v2-0

Shnorhokian, S, Mitri, HS \& Moreau-Verlan, L 2014, 'Assessment of stope sequence alternatives in a diminishing ore pillar', in M Hudyma \& Y Potvin (eds), Proceedings of the Seventh International Conference on Deep and High Stress Mining, Australian Centre for Geomechanics, Perth, pp. 471-484.

Wiles, T 2017, Map3D, Map3D International Ltd, viewed 5 July 2017, https://www.map3d.com/. 
